\title{
ON THE INVARIANCE OF $q$-CONVEXITY AND HYPERCONVEXITY UNDER FINITE HOLOMORPHIC SURJECTIONS
}

\author{
NGUYEN VAN KHUE AND LE VAN THANH
}

\begin{abstract}
In this note we have proved that 0-convexity and hyperconvexity are invariant under finite holomorphic surjections. Invariance of cohomological $q$ convexity for the case of finite dimension also has been established.
\end{abstract}

It is known [7] that Steinness is invariant under finite holomorphic surjections. In this note we investigate the invariance property for 0-convexity generally, for cohomological completeness, for cohomological $q$-convexity of finite dimension, and for hyperconvexity.

1. The invariance of 0 -convexity. We recall that a complex space $X$ is called $q$-convex if there exists an exhaustion function $\varphi$ on $X$ which is strictly $q$-pseudoconvex outside some compact $K \subset X$. It is known [2] that $X$ is 0 -convex if and only if there exists a proper holomorphic map $\theta$ from $X$ onto a Stein space $S$ such that $\theta_{*} \mathcal{O}_{X} \simeq \mathcal{O}_{S}$ and $\theta$ is biholomorphic outside some set of the form $\theta^{-1} A$, where $A$ is a finite subset of $S$. The proper surjection $\theta: X \rightarrow S$ with $S$ is Stein, such that $\theta_{*} \mathcal{O}_{X} \simeq \mathcal{O}_{S}$ is said to be the Remmert reduction of $X$. By [2] if $X$ is holomorphically convex then there exists a Remmert reduction. In this section we prove the following theorem.

1.1 TheOREM. Let $\varphi: X \rightarrow Y$ be a finite holomorphic surjective map. Then $Y$ is 0 -convex if and only if $X$ is.

The proof of Theorem 1.1 is based on the following assertion, essentially as in [9].

1.2 Assertion. A complex space $X$ is 0 -convex if and only if $\operatorname{dim} H^{1}(X, \mathscr{S})<\infty$ for every coherent ideal subsheaf $\mathscr{S} \subset \mathcal{O}_{X}$.

Proof. The necessity follows from a theorem of Andreotti-Grauert [1].

Conversely, assume that $\operatorname{dim} H^{1}(X, \mathscr{S})<\infty$ for every coherent ideal subsheaf $\mathscr{S}$ of $\mathcal{O}_{X}$. We have to prove that $X$ is 0 -convex.

First we show that $X$ is holomorphically convex. Let $V=\left\{x_{n}\right\}_{n=1}^{\infty}$ be a discrete sequence in $X$ and let $J_{V}$ denote the ideal subsheaf of $\mathcal{O}_{X}$ associated to $V$. Consider the exact sequence:

$$
0 \rightarrow J_{V} \rightarrow \mathcal{O}_{X} \stackrel{\eta}{\rightarrow} \tilde{\mathcal{O}} V \rightarrow 0
$$

Received by the editors April 3, 1984 and, in revised form, May 6, 1985.

1980 Mathematics Subject Classification. Primary 32F10; Secondary 32H35.

(C)1987 American Mathematical Society $0002-9947 / 87 \$ 1.00+\$ .25$ per page 
By hypothesis and by exactness of the cohomology sequence associated to (1) we get

$$
\operatorname{dim} \mathcal{O}(V) / \operatorname{Im} \eta=\operatorname{dim} C^{\infty} / \operatorname{Im} \eta<\infty .
$$

Let $l_{\infty}(V)$ denote the subspace of $\mathcal{O}(V)$ consisting of bounded functions on $V$. Then $\operatorname{dim} C^{\infty} / l_{\infty}(V)=\infty$. Thus by (2) it follows that $\operatorname{Im} \eta \backslash l_{\infty}(V) \neq \varnothing$. This implies that $\sup \left|f\left(x_{n}\right)\right|=\infty$ for some $f \in \mathcal{O}(X)$. Hence $X$ is holomorphically convex.

Let $\theta: X \rightarrow S$ be the Remmert reduction of $X$. To prove that $X$ is 0 -convex it suffices to show that $\theta: X \backslash K \rightarrow S \backslash \theta(K)$ is injective for some compact set $K \subset X$. For a contradiction suppose there is a discrete set $V=\left\{x_{n}, y_{n}\right\}_{n=1}^{\infty}$ such that $\theta\left(x_{n}\right)=\theta\left(y_{n}\right)$ for every $n \geqslant 1$. For each $n$ let $\sigma_{n} \in \mathcal{O}(V)$ be given by the formula

$$
\sigma_{n}\left(x_{n}\right)=1, \quad \sigma_{n} \mid V-x_{n}=0 \text {. }
$$

Since $\theta_{*} \mathcal{O}_{X} \simeq \mathcal{O}_{S}$ it is easy to see that $\left\{\sigma_{n} \bmod \eta\right\}(n=1,2, \ldots)$ is linearly independent in $\mathcal{O}(V) / \operatorname{Im} \eta$. This contradicts (2). Hence Assertion 1.2 is proved.

1.3 AsSERTION. Let $\theta: X \rightarrow Y$ be an $n$-analytic covering and let $Y$ be normal. Then for every coherent ideal subsheaf $\mathscr{S}$ of $\mathcal{O}_{Y}$ there exists a morphism $Q$ : $\theta_{*} \theta * \mathscr{S} \rightarrow \mathscr{S}$ such that $Q e=\mathrm{id}$, where $e: \mathscr{S} \rightarrow \theta_{*} \theta^{*} \mathscr{S}$ is the canonical injection.

Proof. Let $V \subset Y$ be the branch locus of $\theta$ and let $U \subset Y$ be a Stein open subset of $Y$ on which there exists an exact sequence

$$
\mathcal{O}_{Y}^{q} \rightarrow \mathcal{O O}_{Y}^{p} \stackrel{\eta}{\rightarrow} \mathscr{S} \rightarrow 0
$$

Then the sequence

$$
\theta * \mathcal{O}_{Y}^{q} \rightarrow \theta * \mathcal{O}_{Y}^{p} \stackrel{\tilde{\eta}}{\rightarrow} \theta * \mathscr{S} \rightarrow 0
$$

is also exact. Note that $\boldsymbol{\theta}^{*} \mathcal{O}_{Y}^{m} \simeq \mathcal{O}_{X}^{m}$ for every $m \geqslant 1$.

Consider $\sigma \in H^{0}\left(U, \theta_{*} \theta^{*} \mathscr{S}\right)=H^{0}\left(\theta^{-1}(U), \mathscr{S}\right)$. Since $\theta^{-1}(U)$ is Stein [7] we can find $\beta \in H^{0}\left(\theta^{-1}(U), \theta_{X}^{p}\right)$ such that $\tilde{\eta} \beta=\sigma$. Since $Y$ is normal, the formula $P_{U}(\beta)(z)=(1 / n) \sum_{j=1}^{n} \beta\left(x_{j}\right)$ for $z \in U \backslash V$ where $\theta^{-1}(z)=\left\{x_{1}, x_{2}, \ldots, x_{n}\right\}$, defines an element $P_{U}(\beta) \in \mathcal{O} \mathcal{P}_{(}(U)$. Put

$$
Q_{U}(\sigma)=\eta P_{U}(\beta) .
$$

It is easy to see that $Q_{U}(\sigma)$ is independent of choice of $\beta \in H^{0}\left(\theta^{-1}(U), \mathcal{O}_{X}\right)$, $\tilde{\eta} \beta=\sigma$, and $Q_{U}(\sigma)=\sigma$ for all $\sigma \in H^{0}(U, \mathscr{S})$.

Assume now that

$$
\mathcal{O} q_{Y}^{\prime} \rightarrow \mathcal{O} \underline{Y}^{\prime} \rightarrow \mathscr{S} \rightarrow 0
$$

in another exact sequence on $U$. Then there exists a commutative diagram:

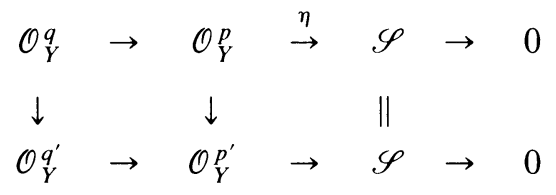

By the commutativity of (8) it follows that $Q_{U}$ is independent of choice of presentation. Hence $Q=\left\{Q_{U}\right\}$ defines a morphism $Q: \theta_{*} \theta^{*} \mathscr{S} \rightarrow \mathscr{S}$ such that $Q e=\mathrm{id}$. Assertion 1.3 is proved. 
Now we are able to prove Theorem 1.1. Assume that $Y$ is 0 -convex. Since $\varphi$ is proper it follows that $X$ is holomorphically convex. Considering the commutative diagram

$\begin{array}{ccc}X & \stackrel{\varphi}{\rightarrow} & Y \\ \theta_{X} \downarrow & & \downarrow \theta_{Y} \\ S_{X} & \stackrel{\tilde{\varphi}}{\rightarrow} & S_{Y}\end{array}$

where $\theta_{X}$ and $\theta_{Y}$ are Remmert reductions of $X$ and $Y$ respectively, it is easy to see that $\theta_{X}$ is biholomorphic outside some compact set $K$ in $X$. Hence $X$ is 0 -convex.

Conversely, assume that $X$ is 0 -convex. We prove that $Y$ is also 0 -convex.

(a) First we consider the case, where $\operatorname{dim} Y<\infty$.

We assume that the theorem has been proved for all complex spaces $Y$ of dimension $<m$. Now assume that $\operatorname{dim} Y=m$. Consider the commutative diagram:

$$
\begin{array}{ccc}
\left(X \times_{Y} \tilde{Y}\right)_{\text {red }} & \stackrel{\tilde{\varphi}}{\rightarrow} & \tilde{Y} \\
\downarrow \eta & & \downarrow \nu \\
X & \stackrel{\varphi}{\rightarrow} & Y
\end{array}
$$

of finite holomorphic surjective maps, where $\tilde{Y}$ is the normalization of $Y$. By the necessary condition already proved, $\left(X \times_{Y} \tilde{Y}\right)_{\text {red }}$ is 0 -convex. On the other hand, since $\tilde{\varphi}$ is finite and $\tilde{Y}$ normal it follows that $\tilde{\varphi}$ is a finite analytic $n$-covering for some $n$ [4]. Thus by 1.2 and 1.3 we infer that $\tilde{Y}$ is 0 -convex. To prove that $Y$ is 0 -convex by 1.2 it suffices to show that $\operatorname{dim} H^{1}(Y, \mathscr{S})<\infty$ for every coherent ideal subsheaf $\mathscr{S}$ of $\mathscr{O}_{Y}$. Let $\tilde{\mathcal{O}}_{Y}$ denote the coherent analytic sheaf of germs of weakly holomorphic functions on $Y$ [4]. Put $\mathscr{D}=\mathcal{O}_{Y}: \tilde{\mathcal{O}}_{Y}$. Note that $\nu_{*} \mathcal{O}_{\tilde{Y}}=\tilde{\mathcal{O}}_{Y}$ and $\operatorname{supp} \theta_{Y} / \mathscr{D}=N(Y)$ where $N(Y)$ denotes the nonnormal locus of $Y$. Let $\mathscr{V}$ be the coherent ideal subsheaf of $\mathcal{O}_{\tilde{Y}}$ which is the image of $\nu^{*} \mathscr{D} \mathscr{S}=\nu^{-1}(\mathscr{D} \mathscr{S}) \otimes_{\nu^{-1} \mathcal{O}_{Y}} \mathcal{O}_{\tilde{Y}}$ under multiplication. By using the definition of $\mathscr{D}$ it follows that $\nu_{*} \mathscr{V} \subset \mathscr{S}$ and since $\tilde{Y}$ is 0 -convex and $\nu$ is finite we have [4]

$$
\operatorname{dim} H^{1}\left(Y, \nu_{*}, \mathscr{V}\right)=\operatorname{dim} H^{1}(\tilde{Y}, \mathscr{V})<\infty
$$

Since $\nu$ is biholomorphic outside $\nu^{-1}(N(Y))$ it follows that

$$
\operatorname{supp} \mathscr{S} / \nu_{*} \mathscr{V} \subset N(Y) \text {. }
$$

Thus, using the induction hypothesis we get

$$
\operatorname{dim} H^{1}\left(Y, \mathscr{S} / \nu_{*} \mathscr{V}\right)=\operatorname{dim} H^{1}\left(N(Y), \mathscr{S} / \nu_{*} \mathscr{V}\right)<\infty
$$

By (11) and (13) and by the exactness of the cohomology sequence associated to the exact sequence

$$
0 \rightarrow \nu_{*} \mathscr{V} \rightarrow \mathscr{S} \rightarrow \mathscr{S} / \nu_{*} \mathscr{V} \rightarrow 0
$$

we infer that $\operatorname{dim} H^{1}(Y, \mathscr{S})<\infty$.

(b) In the general case, let $Y=\bigcup_{j=1}^{\infty} V_{j}$, where $V$ is an irreducible branch of $Y$ for any $j \geqslant 1$. Since $\tilde{Y}=\bigsqcup_{1}^{\infty} \tilde{V}_{j}$, by the 0 -convexity of $\tilde{Y}$ it is easy to see that there exists $j_{0}$ such that $\tilde{V}_{j}$ is Stein for every $j>j_{0}$. Hence $V_{j}$ is also Stein for every $j>j_{0}$. 
Put

$$
Y_{0}=\bigcup_{j=1}^{j_{0}} V_{j}, \quad Y_{k}=Y_{0} \cup \bigcup_{j=1}^{k} V_{j_{0}+j}
$$

By (a) $Y_{k}$ is 0 -convex for every $k \geqslant 0$.

If $Y_{0}$ is Stein then $Y$ is Stein by [7]. Now we assume that $Y_{0}$ is 0 -convex non-Stein. Thus $Y_{k}$ is 0 -convex non-Stein for every $k \geqslant 0$. Let $\theta_{k}: Y_{k} \rightarrow S_{k}$ be the Remmert reduction of $Y_{k}$. Then we have the following diagram:

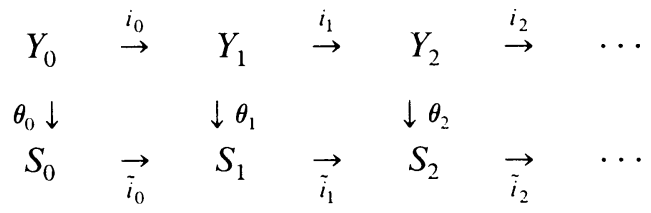

Let $A_{k}$ be a finite subset of $S_{k}$ such that $\theta_{k}: Y_{k}-\theta_{k}^{-1}\left(A_{k}\right) \rightarrow S_{k}-A_{k}$ is biholomorphic. Since $Y_{k}$ non-Stein, $\theta_{k}^{-1}(y)$ is connected of positive dimension for every $y \in A_{k}$ [2]. Then, since $U_{j>j_{0}} V_{j}$ is Stein, and $\theta_{k}^{-1}\left(A_{k}\right)$ is compact connected of positive dimension, it follows that

$$
\theta_{k}^{-1}\left(A_{k}\right)=\theta_{0}^{-1}\left(A_{0}\right) \text { for every } k \geqslant 0 .
$$

From (14) it is easy to see that there exists $k_{0}$ such that

$$
\tilde{i}_{k} \text { is proper injective and } \theta_{0}^{-1}\left(A_{0}\right) \subset \operatorname{Int} Y_{k} \text { for every } k=k_{0} .
$$

Put $S=\lim _{\rightarrow} S_{k}$ and $\theta=\lim _{\rightarrow} \theta_{k}: Y \rightarrow S$. By (14)(15) we infer that $\theta$ is proper, $\theta \mid Y \backslash \theta_{0}^{-1}\left(\overrightarrow{\left.A_{0}\right)}\right.$ is biholomorphic, and $\theta_{*} \mathcal{O}_{Y}=\mathcal{O}_{S}$. Moreover, since $S=\underset{\rightarrow}{\lim } \tilde{i}_{k}\left(S_{k}\right)$ where $\tilde{i}_{k}\left(S_{k}\right)$ are Stein closed subspaces of $S$, it follows that $S$ is holomorphically separated and holomorphically convex and thereby $S$ is Stein. Hence $Y$ is 0 -convex. This completes the proof of Theorem 1.1.

The following is an immediate consequence of Theorem 1.1.

1.4 Corollary. A complex space $X$ is 0 -convex if and only if all its irreducible branches, except for finitely many which are 0-convex, are Stein.

1.5 Corollary. Let $\theta: X \rightarrow Y$ be a proper holomorphic surjective map which is finite outside a compact set. Then $X$ is 0 -convex if and only if $Y$ is.

Proof. Assume that $Y$ is 0 -convex. Considering the commutative diagram (9) it is easy to see that $\theta_{X}$ is finite outside a compact set. Hence by the Steinness of $S_{X}$ we infer that $X$ is 0 -convex. Now assume that $X$ is 0 -convex. Consider the commutative diagram (16)

$$
\begin{array}{ccc}
X & \stackrel{\theta}{\rightarrow} & Y \\
\theta_{X} \downarrow & \searrow^{\eta} & \uparrow \theta^{\prime} \\
S_{X} & \stackrel{\leftrightarrow}{*} & X^{\prime}
\end{array}
$$


in which $X^{\prime}$ is the Stein factorization of $X$ for $\theta$, and $\eta, \beta$ are canonical maps and $\theta^{\prime}$ is induced by $\theta$. It is easy to check that $\theta^{\prime}$ is finite, $\beta$ is finite outside a compact set. This implies that $X^{\prime}$ is 0 -convex. By Theorem 1.1 we infer that $Y$ is 0 -convex.

1.6 Corollary. Let $\theta: X \rightarrow Y$ be a proper holomorphic surjective map and $X$ be 0 -convex. Then $Y$ is also 0-convex.

Proof. Considering the commutative diagrams (10) and (16) and by Theorem (1.1), without loss of generality we may assume that $Y$ is normal. Hence $Y$ is holomorphically convex. Consider the commutative diagram:

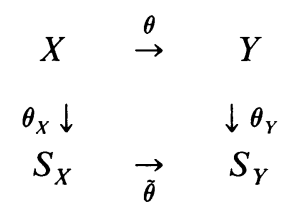

Since $\theta_{X}$ is finite outside a compact set, and $\tilde{\theta}$ is finite, it follows that $\theta_{Y}$ is finite outside a compact set. Hence $Y$ is 0 -convex.

1.7 REMARK. Corollary (1.6) is not true for the holomorphically convex property [7].

2. The invariance of cohomological $q$-completness. A complex space $X$ is called cohomologically $q$-complete (resp. cohomologically $q$-convex) if and only if $H^{p}(X, \mathscr{S})=0$ (resp. $\left.\operatorname{dim} H^{p}(X, \mathscr{S})<\infty\right)$ for every coherent ideal subsheaf $\mathscr{S}$ of $\mathcal{O}_{X}$ and for every $p>q$.

In this section we prove the following theorem.

2.1 THEOREM. Let $\varphi: X \rightarrow Y$ be a finite holomorphic surjective map. Then $X$ is cohomologically q-complete if and only if $Y$ is. If, moreover, $\operatorname{dim} X<\infty$ then $X$ is cohomologically q-convex if and only if $Y$ is.

Proof. Since $\varphi$ is finite it follows that if $Y$ is cohomologically $q$-complete (resp. cohomologically $q$-convex) then $X$ is too.

As in the proof of Theorem 1.1(a) it follows that if $\operatorname{dim} X<\infty$ and $X$ is cohomologically $q$-complete (resp. cohomologically $q$-convex), then so is $Y$.

Thus to find the proof of the theorem it suffices to prove the following

2.2 ASSERTION. Let $X=\bigcup_{k=1}^{\infty} X_{k}, X_{k}$ is the union of all irreducible branches of $X$ of dimension $\prec k$. If $X_{k}$ is cohomologically $q$-complete for every $k \geqslant 1$, then $X$ is also cohomologically $q$-complete.

Proof. Let $\mathscr{S}$ be a coherent ideal subsheaf of $\mathcal{O}_{X}$ and $J_{k}=\mathscr{T}_{X_{k}}$-the ideal subsheaf of $\mathcal{O}_{X}$ associated to $X_{k}$. By $\eta_{k}: \mathcal{O}_{X} \rightarrow \tilde{\mathcal{O}}_{X_{k}}$ denotes the canonical map. Put $\mathscr{S}_{k}=\eta_{k}(\mathscr{S})$. Since any open set in $X$ is contained in some $X_{k}$ it follows that

$$
\mathscr{S}=\lim _{\leftarrow}\left\{\mathscr{S}_{k}, \omega_{k}^{j}\right\}
$$

where $\omega_{k}^{j}: \mathscr{S}_{k} \rightarrow \mathscr{S}_{j}$ is a canonical map.

Let $\mathscr{U}$ be a Stein open covering of $X$. By hypothesis we have

$$
H^{p}\left(\mathscr{U}, \mathscr{S}_{k}\right)=H^{p}\left(X, \mathscr{S}_{k}\right)=H^{p}\left(X_{k}, \mathscr{S}_{k}\right)=0
$$


for every $p>q$ and so

$$
\operatorname{Im}\left\{H^{p-1}\left(\mathscr{U}, \mathscr{S}_{k+1}\right) \rightarrow H^{p-1}\left(\mathscr{U}, \mathscr{S}_{k}\right)\right\}=H^{p-1}\left(\mathscr{U}, \mathscr{S}_{k}\right)
$$

for every $p>q$ and $k \geqslant 1$.

Consider $\sigma \in Z^{p}(\mathscr{U}, \mathscr{S}), p>q$. By (17) for each $k \geqslant 1$ we find $\beta_{k}^{\prime} \in$ $C^{p-1}\left(\mathscr{U}, \mathscr{S}_{k}\right)$ such that $\delta^{p-1} \beta_{k}^{\prime}=\eta_{k} \sigma$. Put $\beta_{1}=\beta_{1}^{\prime}$ and consider $\omega_{2}^{1} \beta_{2}^{\prime}-\beta_{1}$. Since $\delta^{p-1}\left(\omega_{2}^{1} \beta_{2}^{\prime}-\beta_{1}\right)=0$, by (18) with $k=1$ we find $\beta_{2}^{\prime \prime} \in Z^{p-1}\left(\mathscr{U}, \mathscr{S}_{2}\right)$ such that

$$
\omega_{2}^{1}\left(\beta_{2}^{\prime \prime}-\beta_{2}^{\prime}\right)+\beta_{1}=\delta^{p-2} \gamma \text { for some } \gamma \in C^{p-2}\left(\mathscr{U}, \mathscr{S}_{1}\right) \text {. }
$$

Since $\mathscr{U}$ is a Stein open covering, there exists $\tilde{\gamma} \in C^{p-2}\left(\mathscr{U}, \mathscr{S}_{2}\right)$ such that $\omega_{2}^{1} \tilde{\gamma}=\gamma$. Put

$$
\beta_{2}=-\beta_{2}^{\prime \prime}+\beta_{2}^{\prime}+\delta^{p-2} \tilde{\gamma} .
$$

Then $\delta^{p-1} \beta_{2}=\eta_{2} \sigma$ and $\omega_{2}^{1} \beta_{2}=\omega_{2}^{1}\left(\beta_{2}^{\prime}-\beta_{2}^{\prime \prime}\right)+\omega_{2}^{1} \delta^{p-1} \tilde{\gamma}=\omega_{2}^{1}\left(\beta_{2}^{\prime}-\beta_{2}^{\prime \prime}\right)+\delta^{p-1} \omega_{2}^{1} \tilde{\gamma}$ $=\omega_{2}^{1}\left(\beta_{2}^{\prime}-\beta_{2}^{\prime \prime}\right)+\beta_{1}+\omega_{2}^{1}\left(\beta_{2}^{\prime \prime}-\beta_{2}^{\prime}\right)=\beta_{1}$. Continuing this process we get a sequence $\left\{\beta_{n}\right\}$ such that for every $n \geqslant 1$ :

$$
\beta_{n} \in C^{p-1}\left(\mathscr{U}, \mathscr{S}_{n}\right), \quad \delta^{p-1}\left(\beta_{n}\right)=\eta_{n}(\sigma) \quad \text { and } \omega_{n+1}^{n} \beta_{n+1}=\beta_{n} .
$$

Thus $\beta=\left\{\beta_{n}\right\} \in C^{p-1}(\mathscr{U}, \mathscr{S})$ and $\delta^{p-1} \beta=\sigma$. Hence $H^{p}(X, \mathscr{S})=0$ and 2.2 is proved.

The following is an immediate consequence of Theorem 2.1.

2.3 Corollary. $X$ is cohomologically q-complete if and only if every irreducible branch of $X$ is.

3. The invariance of the hyperconvexity. We recall that a Stein space $X$ is called hyperconvex (resp. strongly hyperconvex) if there exists a plurisubharmonic (resp. strictly plurisubharmonic) negative exhaustion function on $X[8]$. In this section the following theorem is proved.

3.1 TheOREM. Let $\theta: X \rightarrow Y$ be a finite holomorphic surjective map of finite-dimensional complex spaces. Then:

(i) If $Y$ is strongly hyperconvex having a strictly plurisubharmonic negative exhaustion $C^{2}$-function, then $X$ is strongly hyperconvex.

(ii) If $Y$ is irreducible and $X$ is strongly hyperconvex having a strictly plurisubharmonic negative exhaustion $C^{2}$-function, then $Y$ is strongly hyperconvex.

We need the following.

3.2 Lemma. If $X$ is strongly hyperconvex and $Y$ is normal, then so is $Y$.

Proof. Let $\psi$ be a strictly plurisubharmonic negative exhaustion function of $X$. By the integer lemma [4] we infer that $\theta: X \rightarrow Y$ is an analytic covering. Thus we can define a function $\varphi$ on $Y$ by the formula

$$
\varphi(y)=\operatorname{Tr}_{\theta}(\psi)(y)=\sum_{\theta x=y} \psi(x)
$$

(the points of $\theta^{-1}(y)$ being counted with the right multiplicity). 
Since $\psi<0$ it follows that $\varphi$ is an exhaustion function. First we prove that $\varphi$ is plurisubharmonic. By a theorem of Fornaess and Narasimham [5] it suffices to show that $\varphi \sigma$ is subharmonic for any holomorphic map $\sigma$ of unit disc $D \subset C$ into $Y$.

Given such a map $\sigma: D \rightarrow Y$, consider the commutative diagram:

$$
\begin{array}{ccc}
\left(D \times{ }_{Y} X\right)_{\mathrm{red}} & \stackrel{\tilde{\sigma}}{\rightarrow} & X \\
\tilde{\theta} \downarrow & & \downarrow \theta \\
D & \rightarrow & Y
\end{array}
$$

in which $\theta$ and $\tilde{\theta}$ are analytic coverings. It is easy to see that the branching order $O_{\tilde{\theta}}(x)=O_{\tilde{\theta}}(\sigma x)$ for any $x \in\left(D \times_{Y} X\right)_{\text {red }}$. Thus $\left(\operatorname{Tr}_{\theta} \psi\right) \sigma=\operatorname{Tr}_{\tilde{\theta}}(\psi \tilde{\sigma})$. Hence it remains to show that $\operatorname{Tr}_{\tilde{\theta}}(\psi \tilde{\sigma})$ is subharmonic. The problem is local on $D$, whence, without loss of generality, we can assume that there exists an embedding $e$ : $\left(D \times{ }_{Y} X\right)_{\text {red }} \rightarrow C^{n}$ for some $n$. Then we have the commutative diagram:

$$
\begin{array}{ccc}
\left(D \times_{Y} X\right)_{\mathrm{red}} \stackrel{\tilde{e}=(\tilde{\theta}, e)}{\rightarrow} & D \times \mathbf{C}^{n} \\
& \tilde{\theta} \searrow & \downarrow \tilde{\pi} \\
& & D
\end{array}
$$

in which $\tilde{\pi} \mid A: A \rightarrow D, A=\tilde{e}\left(D \times_{Y} X\right)_{\text {red }}$, is an analytic covering. Since

$$
\left.\operatorname{Tr}_{\tilde{\theta}}(\psi \tilde{\boldsymbol{\sigma}}) \circ \tilde{e}^{-1}\right|_{A}=\operatorname{Tr}_{\tilde{\pi}}\left(\left.\psi \circ \tilde{\boldsymbol{\sigma}} \circ \tilde{e}^{-1}\right|_{A}\right),
$$

the subharmonicity of $\operatorname{Tr}_{\tilde{\theta}}(\psi \tilde{\sigma})$ follows from a lemma of [5].

If $\sigma$ is a $C^{2}$-function on a neighborhood $V$ of a point $y_{0} \in Y$ such that partial derivatives of order $\leqslant 2$ have sufficiently small absolute values, then $\psi+\sigma \theta$ is plurisubharmonic. Since $\operatorname{Tr}_{\theta}(\psi)+\sigma=\operatorname{Tr}_{\theta}(\psi+\sigma \theta)$ we infer that $\operatorname{Tr}_{\theta}(\psi)+\sigma$ is plurisubharmonic. Thus $\operatorname{Tr}_{\theta} \psi$ is strictly plurisubharmonic by definition. The lemma is proved.

3.3 Lemma. If $Y$ is irreducible and $\tilde{Y}$ is strongly hyperconvex, then so is $Y$.

Proof. Since $Y$ is irreducible, the normalization map $\nu: \tilde{Y} \rightarrow Y$ is homeomorphic. Thus $\psi \circ \nu^{-1}$ is a continuous negative exhaustion function on $Y$, where $\psi$ is that function on $Y$. Since for every holomorphic map $\sigma: D \rightarrow Y$ the map $\nu^{-1} \sigma$ is holomorphic, as in the proof of the Lemma 3.2 we infer that $\psi \nu^{-1}$ is strictly plurisubharmonic. Hence $Y$ is strongly hyperconvex.

ProOF OF THEOREM 3.1. (i) Let $\varphi$ be a strictly plurisubharmonic negative exhaustion $C^{2}$-function on $Y$. We can assume that $Y$ is embedded in $C^{n}$ for some $n$. It is known [6] that there exists a relatively compact Stein open covering $\left\{U_{j}\right\}$ of $C^{n}$ of finite order and a $C^{\infty}$-partition $\left\{\rho_{j}\right\}$ of unity subordinate to $\left\{U_{j}\right\}$ such that $\left|D^{a} \rho_{j}(x)\right| \leqslant C_{\alpha}$ for all $\alpha$ and for all $j$. Since $\theta^{-1}\left(U_{j}\right)$ is a relatively compact Stein open set, we may find a strictly plurisubharmonic nonnegative $\psi_{j} C^{\infty}$-function on $\theta^{-1}\left(U_{j}\right)$. We set

$$
\psi(x)=\sum_{j} \rho_{j}(\theta x) \psi_{j}(x)+\varphi(\theta x)
$$


By calculating $\partial^{2} \psi / \partial z \partial \bar{z}$ (in the local coordinate of $X$ ) we conclude that in choosing $\psi_{j}$ such that the absolute values of their partial derivatives of order $\leqslant 2$ is sufficiently small, $\psi(x)$ is a strictly plurisubharmonic negative exhaustion function of $X$. Hence $X$ is strongly hyperconvex.

(ii) Considering the commutative diagram:

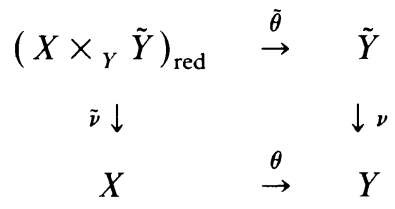

of the finite surjective maps, by (i) and by Lemma 3.2 and 3.3 we get strong hyperconvexity of $Y$. The theorem is proved.

3.4 REMARK. In [3] Diederich and Fornaess have proved that every Stein bounded domain in $C^{n}$ with $C^{2}$-boundary has a strictly plurisubharmonic negative exhaustion $C^{2}$-function.

\section{REFERENCES}

1. A. Andreotti and $\mathrm{H}$. Grauert, Théorèmes de finitude pour la cohomologie des espaces complexes, Bull. Soc. Math. France 90 (1962), 193-259.

2. H. Cartan, Quotients of complex spaces, Contributions to Function Theory, Tata Inst. of Fund. Res., Bombay, 1960, pp. 1-15.

3. K. Diederich and J. E. Fornaess, Pseudoconvex domain: bounded strictly plurisubharmonic exhaustion functions, Invent. Math. 39 (1977), 129-141.

4. G. Fischer, Complex analytic geometry, Springer-Verlag, Berlin and New York, 1976.

5. J. E. Fornaess and R. Narasimhan, The Levi problem on complex spaces with singularities, Math. Ann. 243 (1980), 47-72.

6. B. Malgrange, Ideal of differentiable functions, Oxford Univ. Press, 1966.

7. A. Markoe, Invariant of holomorphic convexity, Math. Ann. 217 (1975), 267-270.

8. R. Narasimhan, A note on Stein spaces and their normalizations, Ann. Scuola Norm. Sup. Pisa 16 (1962), 327-333.

9. __ The Levi problem for complex spaces. II, Math. Ann. 146 (1962), 195-216.

Institut de Mathematiques, P. O. Box 631, Bo Ho, Hanoi, Vietnam 